- A rare case of mental nerve paraesthesia during orthodontic treatment is described.

- Mental nerve paraesthesia can present as the first sign of significant pathology elsewhere in the head and neck region

- The importance of thorough investigation of patients presenting with a history of facial numbness is highlighted.

- Guidelines for referral have been suggested where orthodontic treatment is suspected as the cause.

\title{
Temporary mental nerve paraesthesia secondary to orthodontic treatment - a case report and review
}

\author{
P. J. Willy, ${ }^{1}$ P. Brennan ${ }^{2}$ and J. Moore ${ }^{3}$
}

Patients complaining of facial paraesthesia may present to the dental practitioner. The differential diagnosis includes malignant neoplastic disease and therefore warrants prompt hospital referral. Mental nerve paraesthesia during orthodontic treatment is a very rare occurrence. A case of mental paraesthesia during fixed upper and lower orthodontic treatment is presented. This report highlights the importance of thorough investigation of patients presenting with a history of facial numbness, and provides a review of the aetiology and management of this problem for the dental practitioner. Guidelines for referral have been suggested where orthodontic treatment is suspected as the cause.

Neuropathies can affect a single nerve (mononeuropathy) or several nerves (polyneuropathy) and result in sensory, motor and/or autonomic deficits in the affected region. Causes of cranial neuropathies can be classified as intracranial or extracranial. Intracranial causes include stroke, transient ischaemic attack and tumours. Extracranial causes can include trauma, malignancy (which can be primary, ${ }^{1}$ metastatic $^{2}$ or haematological ${ }^{3}$ in origin) and infection. Iatrogenic causes of altered trigeminal nerve function include radiotherapy, chemotherapy and mandibular surgery.

In a general dental practice setting, a patient presenting with sudden onset of facial paraesthesia is an unusual occurrence. Although the inferior dental nerve (IDN) is relatively protected by its tunnelled course through the bony mandible there is

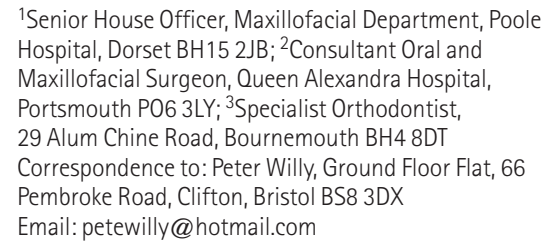

๑) British Dental Journal 2004; 196: 83-84 still potential lower lip sensation to be altered. Reported dental causes of lower lip paraesthesia include third molar extractions, surgical molar/premolar extractions, implant placement, needle trauma following IDN block, periapical inflammation, lower denture compression of the mental nerve and neural injury following endodontic treatment. Mental nerve paraesthesia resulting from orthodontic treatment is very rare and only four cases have been previously described. ${ }^{4-6}$ This report describes a further case of isolated mental nerve paraesthesia during orthodontic treatment. The investigation and management of this phenomenon - which can be the first manifestation of significant underlying disease, is discussed.

\section{CASE REPORT}

A fit and well 17-year-old male undergoing orthodontic treatment was referred with gradual onset of left mental nerve paraesthesia. The orthodontic treatment had been with pre-adjusted edgewise fixed appliances in both arches over the previous 15 months for a crowded Class II Division 2 malocclusion on a skeletal I base. The patient complained of a diffuse dull ache in the left side of the mandible for 2 weeks following the placement of a lower .020 steel aligning archwire. This resolved gradually over 5 days but left the patient with decreased sensation on the left side of his chin. The area of paraesthesia had gradually spread superiorly to involve the left side of the lower lip as well as chin. The lower archwire was made passive and the patient referred as a precaution for further investigation.

Clinical examination revealed decreased light touch and pin prick sensation confined to the left vermilion and upper region of the lower lip combined with paraesthesia of the left side of the chin. The teeth, gingivae and tongue were not affected. Sensation of the skin overlying the left side of the inferior mental symphysis was normal. Maxillary and ophthalmic divisions of the trigeminal nerve as well as all other cranial nerves were normal on both sides. General neurological examination revealed no further deficits in either sensation or motor function.

Intra-oral examination was uneventful. Dental panoramic tomography (Fig. 1) showed the mental foramen lying immediately below the apex of the LL5 (35). The apices of the molar teeth on the left side of the mandible did not encroach on the inferior dental canal. The LL5 (35) was slightly mesially angulated and had therefore been subjected to some uprighting movement with levelling of the occlusal plane.

The patient underwent magnetic resonance imaging (axial and coronal T1 and 
T2 weighted images) which excluded pathology in the upper neck, infratemporal fossa, base of skull and intra-cranially. There was evidence of inflammation in the right maxillary antrum consistent with sinusitis. Unfortunately, it was not possible to visualise the IDN in the mandibular canal as the images of both the mandible and maxilla were obscured by an artefact from the fixed appliance metalwork. At review 3 weeks later (4 weeks following previous archwire adjustment), the sensation in the chin and lip had returned to normal.

After the symptoms of numbness had resolved, treatment was resumed uneventfully. One year later there had been no recurrence of paraesthesia.

\section{DISCUSSION}

The mylohyoid and lingual nerves branch from the main trunk of the mandibular nerve before it pierces the mandible. Sensation of the tongue, lingual mucosa and skin overlying the lower mental symphysis was normal. The area of paraesthesia in this case was limited to the lower lip and chin only. Disruption of nerve function appeared to originate close to the mental foramen as sensation in the labial gingiva and vitality testing was normal.

The mental foramen usually lies directly below the anatomical crown of the mandibular second premolar and is situated around 60\% of the distance from the second premolar buccal cusp tip to the inferior border of the mandible. The mental foramen is intersected by the long axis of the lower second premolar in only $11 \%$ of cases, in the remainder there is an approximate 50:50 split where the foramen lies either anterior or posterior to this long axis. ${ }^{7}$ Bucco-lingually the IDN runs in contact with the lingual plate in the ramus and usually stays close to the lingual plate throughout the mandible before turning sharply to traverse the body of the mandible in the premolar region and exit the mental foramen. ${ }^{8}$

Carter and Keen described three distinct anatomical patterns of the intramandibular course of the IDN: type $1-$ the IDN courses straight towards the mental foramen lying close to the root apices; type 2 - the nerve follows a lower pathway in the mandible with dental branches passing obliquely in an antero-superior direction to the teeth; and type 3 where the IDN divides into two branches, one passing superiorly to supply the molar teeth and the other inferiorly to innervate the premolars, canines and incisors. ${ }^{9}$

In another study the main trunk of the IDN gave branches to the molar and premolar teeth immediately prior bifurcating into the mental and incisive branches in

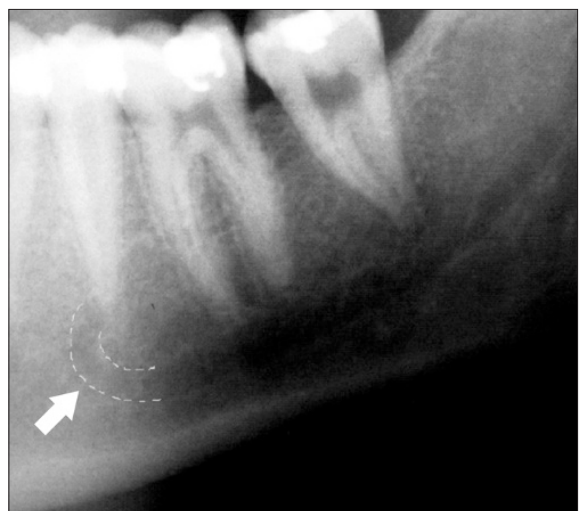

Fig. 1 Close up of a dental panoramic tomogram showing the proximity of the left second premolar apex to the mental foramen. The inferior alveolar nerve has been highlighted

the molar region. A plexus innervated the premolar teeth with origins from the main trunk, molar and incisive branches. ${ }^{10}$ Others have found that the anatomy of the IDN is variable, however in the majority of cases there is a plexus of nerves above the main nerve trunk which supply sensation to the teeth. ${ }^{8}$

In the patient described in this report a type 1 IDN was present according to Carter and Keen's classification. ${ }^{9}$ Visualisation of the bucco-lingual position of the IDN from the MRI scan was not possible because of an artefact from the orthodontic metalwork. From the DPT, the mental foramen was positioned immediately below the long axis of the second premolar and the apex was closely related to the mental foramen. There was normal sensation in the teeth and gingivae suggesting that the cause of paraesthesia was compression of the mental nerve close to the mental foramen.

To the authors' knowledge, only four cases of lower lip paraesthesia during orthodontic treatment have been previously reported. Two of these cases were attributed to the mandibular second premolar and two to the mandibular second molar. Stirrups presented two cases where the lower second premolar crown had been moved in a buccal direction resulting in mental paraesthesia. ${ }^{4}$ It was postulated that the ID canal ran lingual to the apices of these teeth hence tooth movement caused pressure on the neurovascular bundle. Krogstad et al. found that a lower second molar tooth root was intimately related to a lingually positioned ID canal with resultant mental paraesthesia. ${ }^{5}$ Tang et al. found that intrusion of the lower second molar tooth following placement of a heavy rectangular archwire was the probable cause. ${ }^{6}$ In all cases the paraesthesia resolved within 2 days to 4 weeks of the archwire being removed or the relevant tooth being extruded. In three of these cases the onset of paraesthesia occurred following an archwire change.
Isolated cranial nerve neuropathy can present as the first symptom of intracranial or extra-cranial focal lesions. The persistence of symptoms for warrants prompt referral for specialist investigation and management, even if clinical examination finds the neurological deficit confined to one branch. Where orthodontic treatment is a potential cause, symptoms lasting more than 2 weeks following appliance deactivation require further specialist investigation. The presence of any metalwork (orthodontic or restorative) will cause radiation scatter and image artefact with both CT and MRI scanning. In this case the removal of the orthodontic brackets prior to scanning would have enabled the proximity of the inferior alveolar nerve to the lower second premolar apex to be assessed in three dimensions.

\section{CONCLUSION}

Although mental nerve paraesthesia during orthodontic treatment is rare, it may be caused by many other processes. As the differential diagnosis of trigeminal nerve paraesthesia includes malignant neoplasms, patients presenting in the dental setting with previously undiagnosed cranial nerve neuropathies should be referred immediately. Where orthodontic treatment is a possible cause appliances should first be inactivated. If symptoms have not resolved after 2 weeks specialist referral for further investigation should be instigated.

The authors would like to thank Mr A. F. Markus, Consultant Oral and Maxillofacial Surgeon, for allowing us to report this case. We are also grateful to the medical photography department at Poole Hospital for their help.

1. Raveh $T$, Neuman $A$ R, Weinberg A, Alfeie M, Moor EV Caspi R, Lipton H A, Wexler M R. Treatment of extensive malignant schwannoma of the mandibular nerve. Ann Plast Surg 1995; 34: 637-641.

2. Laurencet F M, Anchisi S, Tullen E, Dietrich PY. Mental neuropathy: report of five cases and review of the literature. Crit Rev Oncol Hematol 2000 ; 34: 71-79.

3. Ojanguren J M, Garcia-Monco C, Capelastegui A, Matinez C, Atutxa K, Carrascosa T. Numb chin syndrome in four patients with haematological malignancies. Haematolica 1999 ; 84: 952-953.

4. Stirrups D R. Temporary mental paraesthesia: an unusual complication of orthodontic treatment. BrJOrthod 1985; 12: 87-89.

5. Krogstad O, Omland G. Temporary paraesthesia of the lower lip: a complication of orthodontic treatment. A case report. Br J Orthod 1997; 24: 13-15.

6. Tang N C, Selwyn Barnett C L, Bligh SJ. Lip paraesthesia associated with orthodontic treatment - a case report. BrDent J 1994 ; 176: 29-30.

7. Phillips J L, Weller R N, Kulild J C. The mental foramen Part 1. Size, orientation and positional relationship to the mandibular second premolar. J Endodont 1990; 16: 221-223.

8. Gowgiel J M. The position and course of the mandibular canal. J Oral Implantol 1992; 18: 383-85.

9. Carter R B, Keen E N. The intramandibular course of the inferior alveolar nerve. J Anat 1971; 108: 433-440.

10. Wadu S G, Penhall B, Townsend G C. Morphological variability of the human inferior alveolar nerve. Clin Anat 1997; 10: 82-87. 